\title{
Fault Detection and Isolation for Brake Rotor Thickness Variation
}

\author{
Xinyu $\mathrm{Du}^{1}$, Lichao $\mathrm{Mai}^{1}$, Hamed Kazemi ${ }^{2}$ Hossein Sadjadi ${ }^{2}$ \\ ${ }^{1}$ General Motors Research \& Development, Warren, MI, 48092, USA \\ xinyu.du@gm.com \\ lichao.mai@gmail.com \\ ${ }^{2}$ General Motors Canadian Technical Center, Markham, Ontario, L3R 4H8, Canada \\ hamed.kazemi@gm.com \\ hossein.sadjadi@gm.com
}

\begin{abstract}
Brake rotors are critical parts of the disc braking system for modern vehicles. One common failure for brake rotors is the thickness variation, which may result in unpleasant brake pulsation, vehicle vibration during braking, or eventually lead to the malfunction of the braking system. In order to improve customer satisfaction, vehicle serviceability and availability, it is necessary to develop an onboard fault detection and isolation solution. In our previous work, the vibration features of master cylinder pressure, vehicle longitudinal acceleration and wheel speed were identified as fault signatures. Based on these fault signatures, a vibrationbased fault detection and isolation algorithm is developed in this work. The difference of frequency response between the braking period and the normal driving period (non-braking) is employed to improve the algorithm robustness. The experiment results demonstrate the proposed algorithm can robustly diagnose the thickness variation fault and isolate the fault to each vehicle corner.
\end{abstract}

\section{INTRODUCTION}

The thickness variation of brake rotors, also called disc thickness variation (DTV), is a common fault for the disc brake system. When the fault occurs, a non-uniform braking torque is applied to the vehicle during braking. It is perceived by drivers as brake pulsation or steering wheel vibration. Such a vibration may cause driver's discomfort, or even anxiety about the vehicle safety and quality. For fleet vehicles, the fault is then manually diagnosed by service technicians, which is slow and costly, since the variation of the rotor surface is generally less than $1 \mathrm{~mm}$. Therefore, there is a strong need to develop an automatic diagnostic solution for rotor thickness variation.

Xinyu Du et al. This is an open-access article distributed under the terms of the Creative Commons Attribution 3.0 United States License, which permits unrestricted use, distribution, and reproduction in any medium, provided the original author and source are credited.
Visual inspection is the most common method for the brake rotor diagnostics, which can be performed during vehicle maintenance. To detect the rotor thickness variation, the laser measurement technique is one of the effective approaches to directly measure the rotor surface. A diagnostic tool using holographic interferometry is proposed (Beeck \& Hentschel, 2000). Holographic interferometry is a type of interferometric approach, which measures the rough surface for static and dynamic displacements of an object. For brake rotor diagnostics, it can be used to generate the contour of the brake rotors surfaces. By observing the images generated, the faulty brake rotors with uneven surfaces can be detected.

The visual inspection cannot be performed onboard while the vehicle is in operation. To address this issue, some vibrationbased approaches are used for brake rotor diagnostics and prognostics. The support vector machine (SVM)-based approach is employed (R.Jegadeeshwaran, 2015).Vehicle vibration signals for healthy and faulty brake rotors during braking, are collected using piezoelectric type accelerometers. Vehicle condition labels and the statistical features of the accelerometer measurements, such as standard deviation, variance and kurtosis, are classified by SVM. The faulty brake rotors can then be diagnosed with the trained SVM. The Clonal Selection Classification (CSC) algorithm using the same vibration signals is proposed for rotor diagnostics (Jegadeehwaran \& Sugumaran, 2014). The vehicle vibration signals are collected for both good and faulty brake rotors. The statistical feature sets of vibration signals, including standard error, kurtosis and skewness, are selected and extracted for classification using an attribute evaluator. The selected feature sets for healthy and faulty brake rotors are then classified using the CSC algorithm.

The brake rotor fault can also be detected using the numerical model and the system dynamics theory (Joe, Cha, Sim, Lee, $\&$ Oh, 2008). A linear, lumped and distributed parameters model is set up to perform the stability analysis. The complex eigenvalues of the model represent the dynamic stability of 
the brake system. The analysis of eigenvalues can then be applied to detect the instability of the brake system caused by the faulty brake rotors.

In another study, the noise-based approach is employed for rotor fault diagnostics and prognostics (Ertekin \& Özkurt, 2019). The noise signals from the healthy and faulty brake rotors during braking are collected and processed using the Wavelet Synchro-squeezed Transform. The noise difference between the healthy and faulty brake rotors can be visualized in the scalogram and verified by a quantitative measure of entropy. As a result, the average entropy value of a faulty brake rotor is higher than the value of a healthy rotor. Accordingly, the entropy value can be used as a fault signature to detect faults.

Although the performance of aforementioned approaches is good for certain test datasets, their robustness under different noise factors or other failure modes is still a concern. Furthermore, some sensors required in these approaches are not available in most vehicles. To address these issues, a novel diagnostic approach using existing vehicle signals for brake DTV fault is proposed in this work. As described in our previous work (Kazemi, Du, Dixon, \& Sadjadi, 2019), three vehicle signals are selected to extract fault signatures based on vibration characteristics. A unique fault isolation logic with sophisticated design of enabling conditions and some data processing procedures is used to detect the fault and isolate the fault to each vehicle corner. For the remainder of this report, the details of the fault isolation algorithm are described in section 2 , followed by the validation using the test data from a real vehicle.

\section{BRAKE ROTOR DIAGNOSTIC ALGORITHM}

In this section, the fault diagnostic algorithm for brake rotors is described in detail including the input signals and their enabling conditions, trend removal, order analysis, and fault isolation algorithm.

As described in our previous work (Kazemi, Du, Dixon, \& Sadjadi, 2019), the following vehicle signals are selected: Master Cylinder Pressure (MCP), Longitudinal Acceleration $\left(A_{x}\right)$, Vehicle Speed (VS), Wheel Speed (WS), Brake Pedal Position Gradient (BPPG), and Anti-lock Brake System active (ABSatv). Uneven brake rotor surfaces move the caliper piston in and out during braking, which results in fluid movement in the brake hydraulic system. It is felt all the way in the master cylinder and causes vibration of the master cylinder pressure. The brake pulsation can directly manifest as the vibration of $A_{x}$. VS is collected to determine the vehicle maneuver. BPPG signal is used to determine the brake events. ABSatv is to indicate the anti-lock brake system (ABS) active status, which may cause vehicle vibration even with a healthy rotor. More details about the correlation between these signals and rotor fault severity can be found in our previous work (Kazemi, Du, Dixon, \& Sadjadi, 2019).
In order to exclude the external noises caused by other reasons, e.g. wheel imbalance, rough road, the data is collected both during the normal driving period as well as the braking period. The brake rotor faults are determined if the vibration appears during the braking period but not during the normal driving period. The enabling conditions for the normal driving period are described as below:

$$
\begin{aligned}
& \mathrm{MCP}<0.5 \mathrm{bar} \\
& \mathrm{VS}>27.14 \mathrm{~km} / \mathrm{h} \\
& \mathrm{Ax}>-0.3 \mathrm{~m} / \mathrm{s}^{2}
\end{aligned}
$$

During the normal driving, the MCP values should remain small because the driver does not press the brake pedal. Accordingly, the threshold for MCP is set to 0.5 bar. During braking, especially for hard brakes, the MCP values will increase to 17 bars or even higher (vehicle dependent). When the MCP value is larger than 1 bar, the maneuver may be considered as braking. The threshold of VS is a function of the vibration profile. The vibration frequency is proportional to the vehicle speed during braking. When the vehicle speed is too low, it is difficult to differentiate the vibration due to disc thickness variation (DTV) or pulsating brake caused by the driver. Therefore, the minimum VS value is determined as $27.14 \mathrm{~km} / \mathrm{h}$. The $A_{x}$ values should be positive or slightly smaller than 0 during the normal driving. The $A_{x}$ value less than $-0.3 \mathrm{~m} / \mathrm{s}^{2}$ is considered as the braking maneuver.

In addition to three conditions above, ABSatv and BPPG are employed for enabling conditions during the braking period:

$$
\begin{aligned}
& \mathrm{MCP}>1 \mathrm{bar} \\
& \mathrm{BPPG}<0.5 \% / \mathrm{s} \\
& \mathrm{VS}>27.14 \mathrm{~km} / \mathrm{h} \\
& \text { ABSatv }<0.5
\end{aligned}
$$

The value of ABSatv is either 0 or 1 . The value of ABSatv less than 0.5 indicates that the ABS is not activated. MCP great than 1 bar indicate the brake pedal is pressed. The value of BPPG less than $0.5 \% / \mathrm{s}$ indicates that the position of brake pedal doesn't change too much, and the braking maneuver is in the steady state. No matter which enabling condition is met, the data for MCP, WS, VS and Ax will be saved in the corresponding buffer.

Once the buffer is full, the data are fetched from the buffer. Since only the frequency response at the wheel-speed frequency is of interest, other frequency components, e.g. the slow trend is removed from the data first, which is called trend removal. In the brake buffer, there may exist data from different braking events. The data point at the end of the previous braking event and the data point at the beginning of the current braking event may not be the same. The difference may cause the robustness issue for trend removal. 
Therefore, a variable, named margin, is used to indicate the braking data segment (one braking event). The value of margin is determined by comparing to the previous data type. If the previous data type is braking data, the value is 1 . Otherwise, the value is 0 . The data between two zero values of margin are considered as a braking segment. The length of all data segments is then calculated. The size for the longest segment is saved as max_size.

To remove transient data at the beginning and the end of each braking segment, several data points are removed. The number of points is dynamically determined by the max_size. The bigger the size, the more data points are removed. The difference between the first data point in the selected segment and the last data point in the previous segment is defined as delta. Then all the data in the selected segment are subtracted by delta to remove the difference between the last segment and the current segment.

To complete the trend removal, the moving window size $n$ should be determined from the sampling rate $f_{s}$ and the cutoff frequency $f_{c}$ as follows (Peng, et al., 2019).

$$
n=\frac{\sqrt{0.196202+\left(\frac{f_{c}}{f_{S}}\right)^{2}}}{\frac{f_{C}}{f_{S}}}
$$

In this work, since the vibration frequency for a faulty rotor is about $10-20 \mathrm{~Hz}$, the cutoff frequency is determined as 3 $\mathrm{Hz}$. The sampling rate $f_{s}$ is $100 \mathrm{~Hz}$, so the window size $n$ is 14. The moving average of the data is calculated iteratively by applying the sliding window. The detrended signal is equal to the original data minus the moving average. Data points at the beginning and at the end of the buffer are removed due to inaccurate moving average results.

To demonstrate the effect of trend removal, the MCP signals for a healthy and a faulty rotor are shown in Figure 1, which reflects the difference of the signals before and after the trend

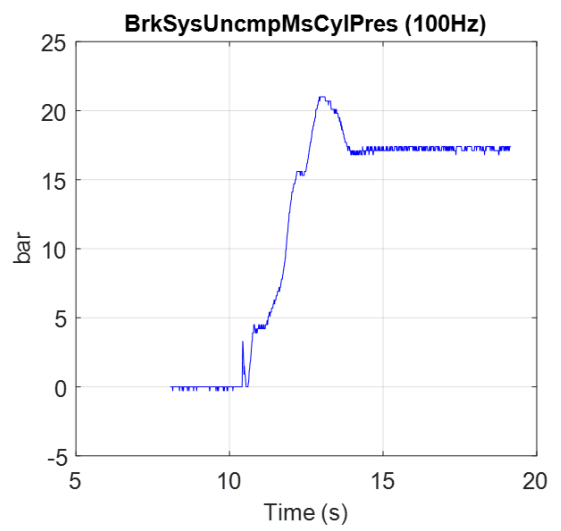

(a)

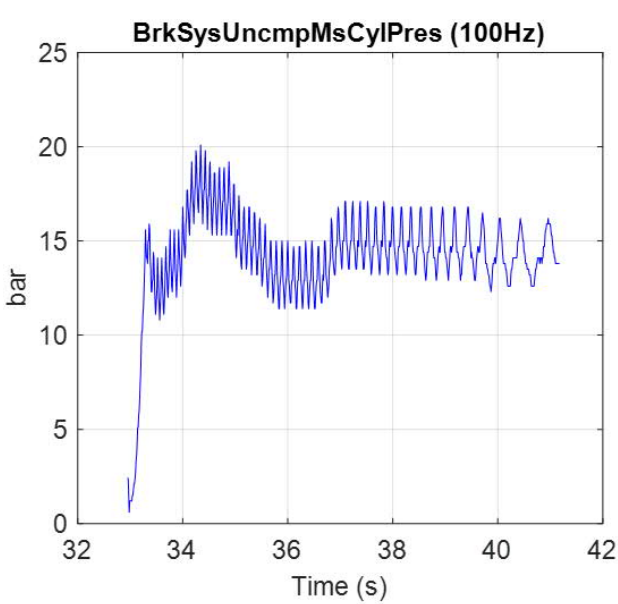

(b)

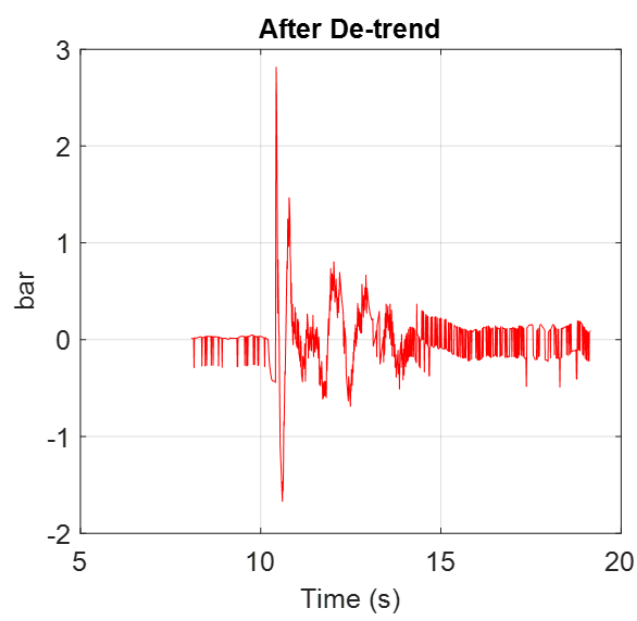

(c)

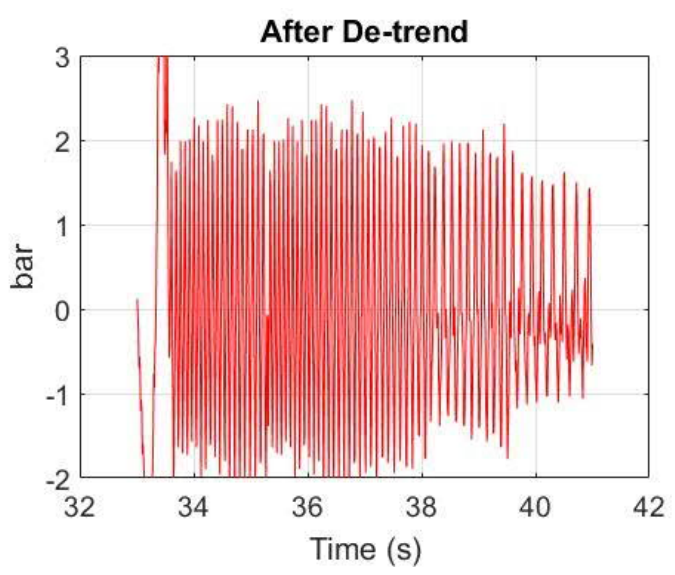

(d)

Figure 1. MCP of: (a) a healthy brake rotor, (b) a faulty brake rotor, (c) a healthy brake rotor after trend removal, and (d) a faulty brake rotor after trend removal. 
removal. It can be observed that the trend removal algorithm can effectively remove the vibration unrelated to DTV, and the vibration amplitude from a faulty rotor is significantly larger than the one from a healthy rotor.

After trend removal, the order analysis is applied to characterize the vibration. When the DTV happens, the 1st order vibration of wheel speed frequency or its harmonic is expected, since the interested signals fluctuate along with the vehicle wheel rotation. In this work, the Fast Fourier Transform (FFT) is used. FFT in a moving window provides the information of the signal, whose frequency components vary over time. The vehicle speed signal is used to determine the frequency order. VS is converted to the rotational frequency from the linear speed $(\mathrm{km} / \mathrm{h})$, using the following equation.

$$
V S(f)=\frac{V S(t)}{2 \pi R \times 3.6}
$$

where $R$ is the radius of the wheel. The margin index generated in the trend removal process is loaded to determine the data segment. The zero margin values in the margin index vector is located as segment end points. The data between each two end points are extracted as a segment. The order analysis is performed on the data in each group, using a fixed window. The size of the window is $w$, which represents the number of data to be processed using FFT. Considering the data size requirement for FFT, the minimum and the maximum values of the fixed window are selected as 32 and 64 , respectively. The VS signal related to the selected data is also segmented to the small groups. If the minimum frequency of selected VS signal is smaller than the cut-off frequency $f_{c}$, which is $3 \mathrm{~Hz}$, the minimum frequency will be replaced with $3 \mathrm{~Hz}$.

Then the selected data are processed using FFT, and the frequency amplitude vectors are generated. To convert the 2sided spectrum to the one-sided form, the first half of the frequency vector is selected, and its values are doubled. The resolution $r$ of the frequency vectors is calculated as the sampling rate $f_{s}$ divided by the fixed window size $\mathrm{w}$ in the segment. The range of the qualified data for the spectrum analysis is defined using the index 1 and index 2, which are calculated using the following equations.

$$
\begin{aligned}
& \text { index } 1=\max \left(\frac{f_{c}-1}{r}, \frac{f_{v, \text { min }}-1}{r}\right) \\
& \text { index } 2=\min \left(\frac{f_{v, \text { max }}+1}{r}, \frac{f_{w, \text { max }}}{r}\right)
\end{aligned}
$$

where, $f_{v, \text { min }}, f_{v, \text { max }}$ are the minimum and the maximum frequencies of the selected VS signals, respectively. $f_{w, \max }$ is the maximum possible frequency of the wheel speed, which is $18 \mathrm{~Hz}$. Then the data between index 1 and index 2 are used to calculate the average amplitude at this frequency. The maximum spectrum for this data segment is saved as $M_{i, \text { max }}$. The average order $M_{i}$ of this segment, is calculated using the following equation.

$$
M_{i}=\frac{M_{i-1} \cdot(i-1)+M_{i, \max }}{i}
$$

where $i$ is the number of the data set selected for the FFT analysis. $M_{i-1}$ is the average order magnitude calculated from the previous datasets.

As shown in Figure 2, when the braking maneuver occurs, the vibrations are more severe for the faulty brake rotor due to the uneven rotor surface. Accordingly, the magnitude of the first order spectrum is higher than that of the healthy brake rotor, which is a good fault signature for fault detection.

The flowchart of the proposed algorithm is shown in Figure 3 , where the thickness variation is detected by monitoring the vibration level of master cylinder, longitudinal acceleration and wheel speed signals. When the vehicle is ignited, the algorithm starts. As described in the previous sections, the vehicle signals including VS, MCP, BPPG, WS and $A_{x}$ are collected and stored in the buffers for the normal driving period and the brake period, respectively based on the enabling conditions. When the number of the data stored in both buffers reaches a value, the MCP data during braking period are detrended using the algorithm described above. Please note that the buffer size or other values mentioned later are calibratable, which are limited by the onboard memory size and computational power.

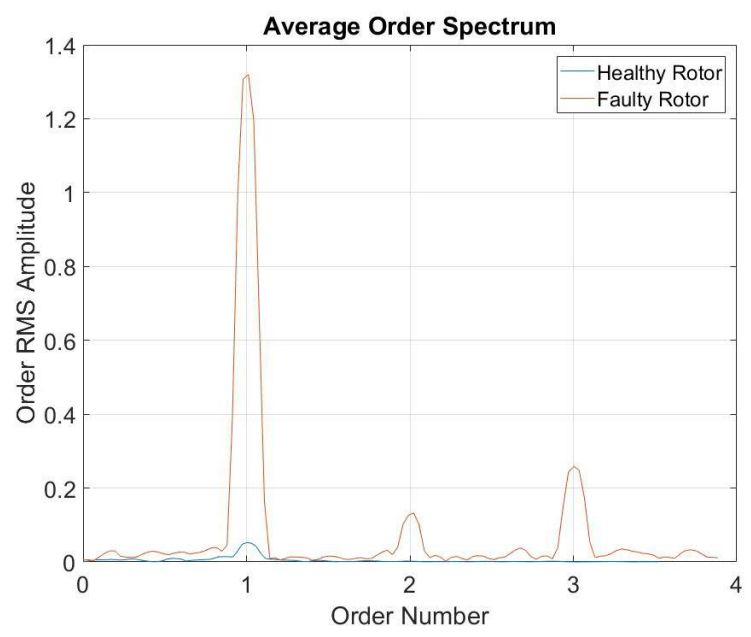

Figure 2. Comparison of MCP average order spectrum of a healthy rotor and a faulty rotor 


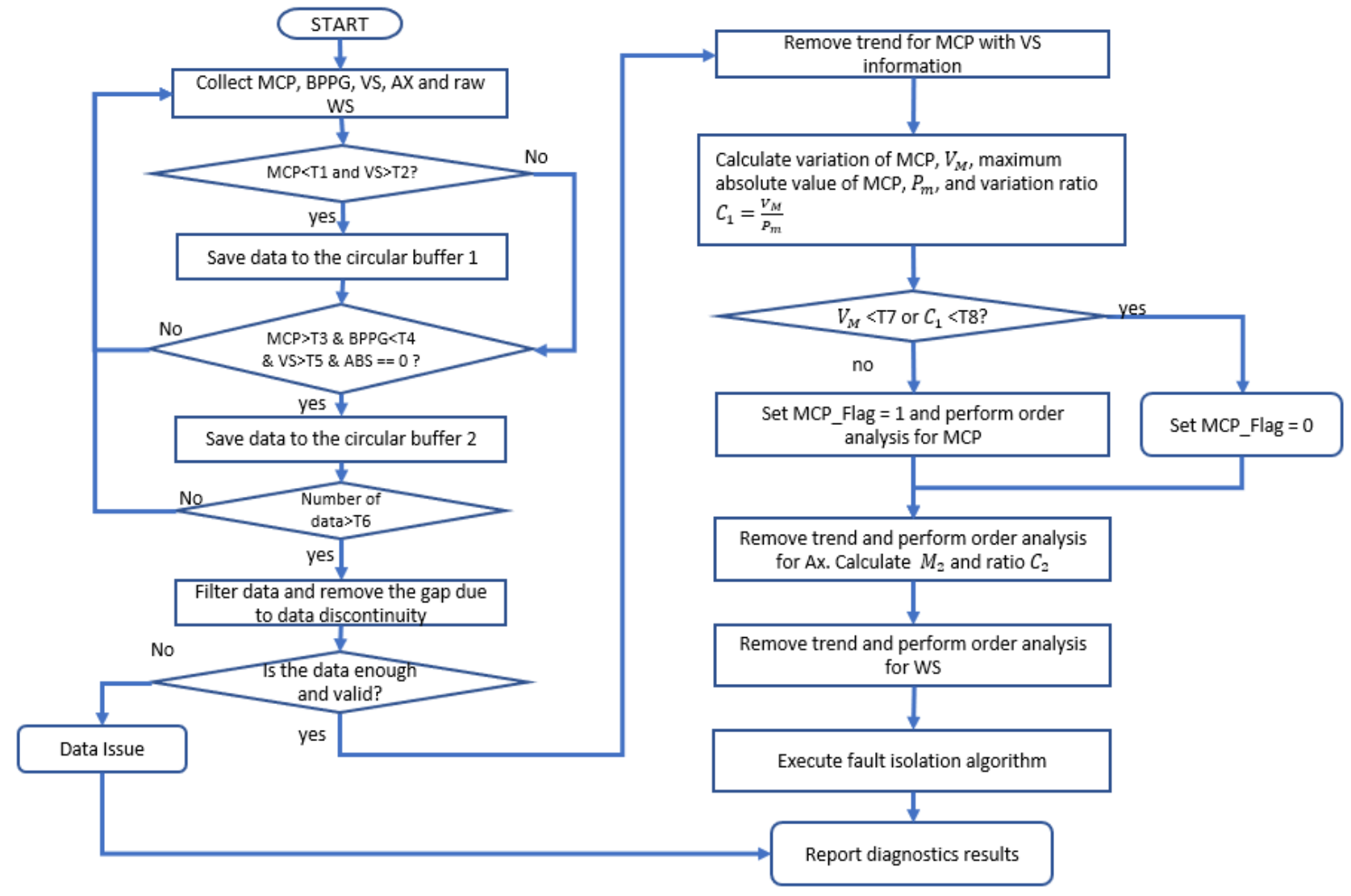

Figure 3. Overall flowchart of the fault detection and isolation algorithm for brake rotors

If the data length of the detrend MCP is less than the threshold, it indicates that there is no enough detrended data, and no diagnostics will be performed. For this situation, the diagnostic result is "data issue".

If the data length is more than the threshold, the variance of the detrended MCP is calculated, denoted as $V_{m}$. And the variance-signal ratio $C_{1}$ is also calculated, which is the variance $V_{m}$ divided by the maximum absolute value of the detrend MCP. If the variance $V_{m}$ is smaller than a threshold or the coefficient $P_{1}$ is smaller than a threshold, the variation of MCP is not severe, and further analysis is performed to determine if the brake rotor is healthy, or it is a rear rotor fault. The threshold of variance or variance ratio is based on healthy data analysis. The value should be validated for each vehicle programs. Please note that the variation of MCP may be small for rear rotor fault due to the isolation of proportioning valve in the braking system. In this situation, the $M C P \_$flag is set as 0 , otherwise, the value is 1 . If the MCP variation is large, the order analysis of the detrend MCP is processed, otherwise, the order analysis of MCP is skipped. Then the signals of $A_{x}$ and WS are detrended, and the order analysis is performed, respectively.

With all the order analysis results, the fault is diagnosed and isolated using the algorithm shown in Figure 4. When the
$M C P \_$flag is equal to 1 , but the order magnitude of MCP, $M_{1}$, is less than a threshold, it is considered as the unknown brake faults. Otherwise, if the order magnitude of $A_{x}, M_{2}$, is smaller than a threshold, it is considered as a "not severe rotor fault". On the other hand, it is considered as a "severe rotor fault".

If the $M C P \_$flag is equal to 0 , and $M_{2}$ is less than a threshold or $C_{1}$ is less than a threshold, the rotor is considered as in the healthy condition. Otherwise, the difference between the order magnitude of $A_{x}$ signals during the normal driving $\left(M_{3}\right)$ and the braking $\left(M_{2}\right)$ is calculated. If the difference is less than a threshold, the brake rotor is considered as healthy.

If the difference is large, or it is a severe fault from the MCP and Ax order analysis, the fault isolation is performed. The difference between the order magnitude for four wheelspeeds during the braking and the normal driving is calculated. The maximum magnitude difference indicates the most severe vibration, which are generated from the faulty brake rotor. All above detection and isolation results are saved to the buffer and can be reported to customers in the end. Since all parameters are related to vehicle vibration levels they may not require further calibration, but may require some validations for different vehicle programs, once it is determined with the initial vehicle program. 


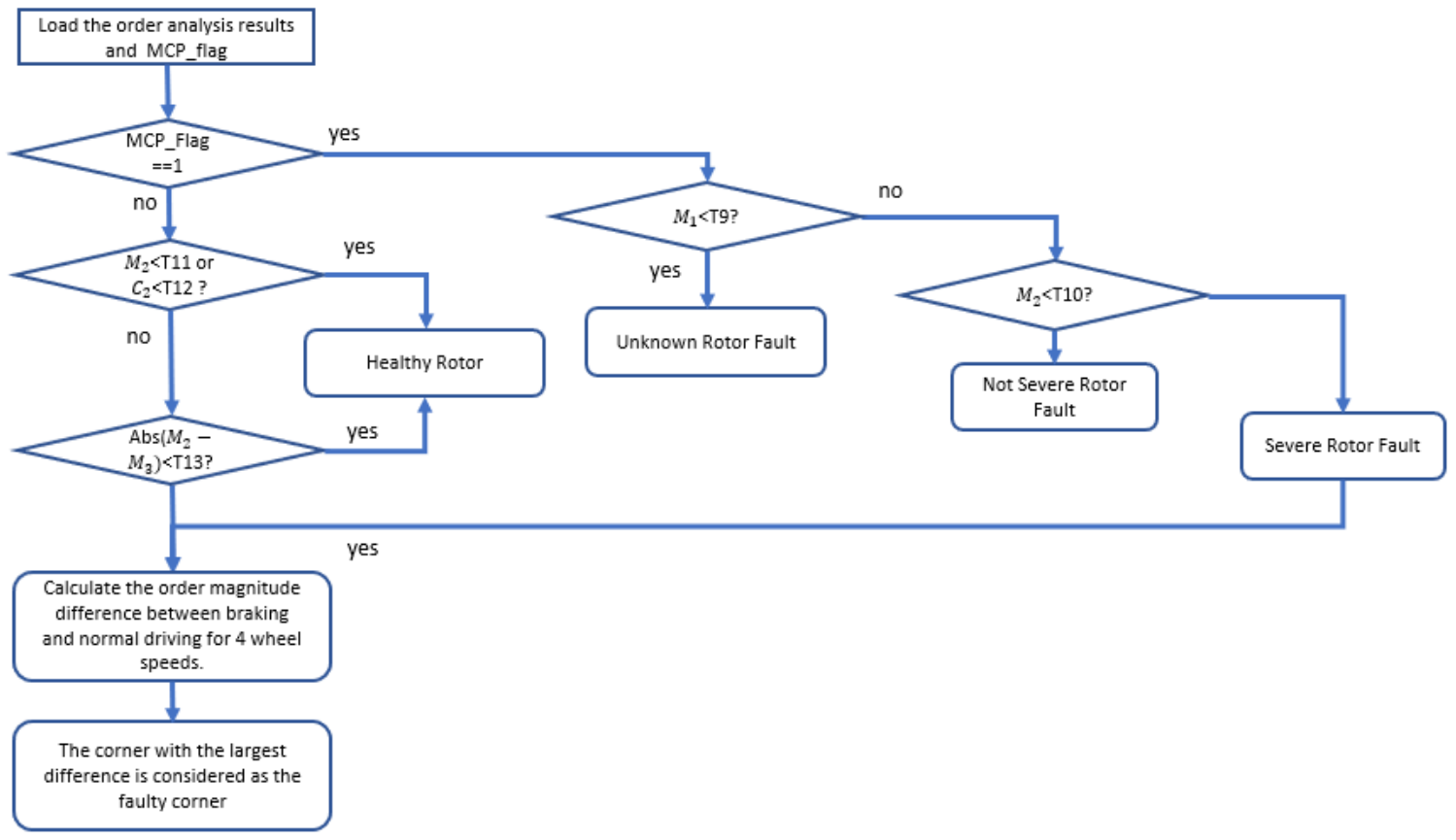

Figure 4. Fault isolation and localization algorithm

\section{VALIDATION}

To verify the proposed method, some vehicle tests were performed with different test conditions.

A 2017 Chevrolet Bolt EV was instrumented for validation. To validate the proposed algorithm, a healthy front rotor was machined with the surface variation of 0.01 inch $(250 \mu \mathrm{m})$, which was used as the faulty rotor. Two healthy rear rotors were machined with the surface variation of 0.01 inch and 0.05 inch, respectively, as the faulty rotors. A total of 859 road tests were performed with the healthy rotor and faulty rotor conditions, shown in Table 1 . To validate the algorithm robustness against other failure modes, 354 tests were performed with a faulty wheel bearing and four healthy brake rotors. All signals were acquired using dSPACE® with the sampling rate of $100 \mathrm{~Hz}$. Each test sample includes at least one braking maneuver. The road tests were performed in several typical driving maneuvers, such as acceleration, deceleration, coast down, cruising and random driving.

For each qualified test, the diagnostic algorithm is activated, and generates a diagnostic result. One typical maneuver example is shown in Figure 5. This maneuver includes acceleration and deceleration. The enabling conditions for brake maneuver are met first, then the vehicle signals are collected and analyzed. The diagnostic results are generated, and the brake rotors faults are detected.

The test results for the front faulty rotors are shown in Figure 6. There are totally 725 test datasets from the healthy rotors and 44 test datasets from the faulty front rotors. After running our algorithms, there is no decision for 305 cases due to the data issue. For the remainder of 464 tests, the algorithm accuracy is $100 \%$, the false positive rate is $0 \%$, and the false negative rate is $0 \%$. For 31 tests with one faulty rotor, our algorithm accurately determine the location to be front left for 30 cases. For the one case that the algorithm is not able to localize the fault, the algorithm output is "not severe fault". The accuracy of the localization algorithm is $96.8 \%$.

The test results for the faulty rear rotors are shown in Figure 7. There were in tola 725 test datasets from healthy rotors and 90 test datasets from faulty rear rotors.

Table 1. Summary of the vehicle road tests

\begin{tabular}{|c|c|c|}
\hline Rotor condition & Location & Num of tests \\
\hline Healthy rotors & N/A & 371 \\
\hline $\begin{array}{c}\text { Healthy rotors with a bad } \\
\text { wheel bearing }\end{array}$ & N/A & 354 \\
\hline $\begin{array}{c}\text { The faulty rotor with 0.01- } \\
\text { inch thickness variation }\end{array}$ & Front left & 44 \\
\hline $\begin{array}{c}\text { The faulty rotor with 0.01- } \\
\text { inch thickness variation }\end{array}$ & Rear left & 38 \\
\hline $\begin{array}{c}\text { The faulty rotor with 0.01- } \\
\text { inch thickness variation }\end{array}$ & Rear left & 52 \\
\hline Total & & 859 \\
\hline
\end{tabular}




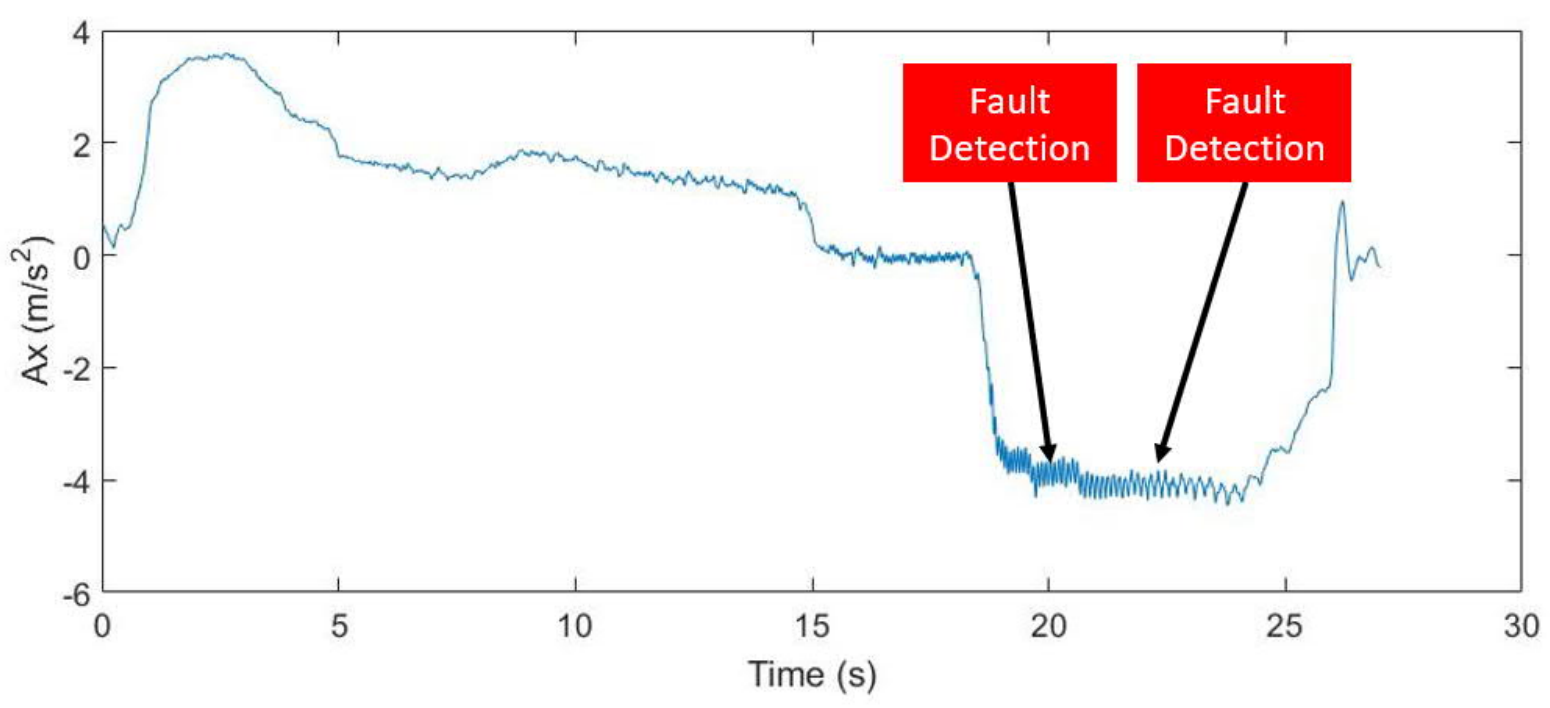

Figure 5. An example of test maneuver

After running our algorithms, there is no decision for 313 cases due to the data issue. For the other 502 tests, the algorithm accuracy is $88.2 \%$, the false positive rate is $0 \%$, and the false negative rate is $85.5 \%$. For all data sets from faulty rotors without data issues, our algorithm accurately determines the location to be rear left. The accuracy of the localization algorithm is $100 \%$.

We observe that the 0.01-inch DTV can be accurately detected in front rotors, but not for rear rotors. 0.05-inch DTV can be captured by algorithms for rear rotors. This is because the rear rotors have less impact on braking system by design to accommodate load transfer. Therefore, the false negative rate is higher for rear rotors. However, with longer time, the algorithm may still be able to catch the fault under certain driving conditions. For all 354 test data sets with 4 healthy rotors and 1 faulty wheel bearing, the algorithm does not generate any false positive alert.

\section{CONCLUSION}

A vibration-based approach is developed in this work to detect and isolate the thickness variation fault for brake rotors. The proposed algorithm can robustly detect the thickness variation fault against other vibration sources by analyzing vehicle signals before and during a brake event. The brake rotor thickness variation can be isolated for the four corners based on comparing the order analysis results of the wheel speed signals. The fault in rear rotors exhibits less vibration in master cylinder pressure than the fault of front rotors. Data from the 725 tests with healthy rotors and 134 tests with faulty rotors using a 2017 Chevrolet Bolt EV show that the accuracy of the proposed algorithm is $100 \%$ for the front rotors and $88.2 \%$ for the rear rotors. Our next focus is to test and improve the proposed algorithms for other failure modes and other noise factors, and to develop the remaining useful life estimation algorithms.

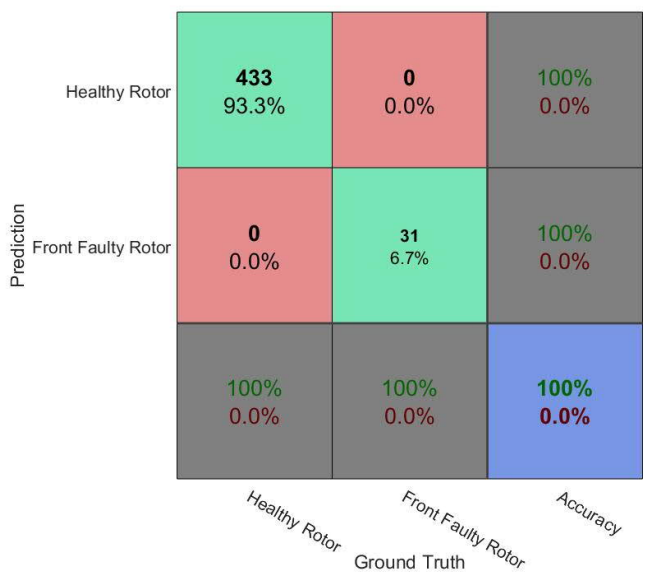

Figure 6. Algorithm performance for front rotors

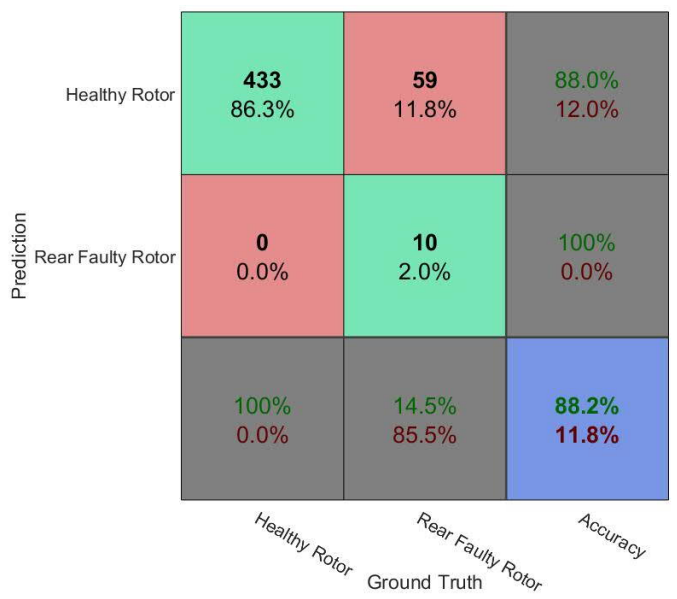

Figure 7. Algorithm performance for rear rotors 


\section{REFERENCES}

Beeck, M. A., \& Hentschel, W. (2000). Laser metrology - a diagnostic tool in automotive development processes. Optics and Lasers in Engineering, 34(2), 101-120.

Ertekin, Z., \& Özkurt, N. (2019). Noise analysis of air disc brake systems using wavelet synchro squeezed transform. Celal Bayar Üniversitesi Fen Bilimleri Dergisi, 15(4), 409-414.

Jegadeehwaran, R., \& Sugumaran, V. (2014). Brake fault diagnosis using Clonal Selection Classification Algorithm (CSCA) - A statistical learning approach. Engineering Science and Technology, 18.

Joe, Y. -G., Cha, B. -G., Sim, H. -J., Lee, H. -J., \& Oh, J. -E. (2008). Analysis of disc brake instability due to friction-induced vibration using a distributed parameter model. International Journal of Automotive Technology, 9, 161-171.

Kazemi, H., Du, X., Dixon, R., \& Sadjadi, H. (2019). A prognostics model to predict brake rotor thickness variation. Proceedings of the Annual Conference of the PHM Society, 11(1).

Peng, Y., Wong, C., Wang, Z., Wan, F., Vai, M. I., Mak, P. U., .. . Rosa, A. C. (2019). Fatigue evaluation using multi-scale entropy of EEG in SSVEP-Based BCI. IEEE Access, 108200-108210.

R.Jegadeeshwaran, V. (2015). Fault diagnosis of automobile hydraulic brake system using statistical features and support vector machines. Mechanical Systems and Siganl Processing, 52-53, 436-446.

\section{BIOGRAPHIES}

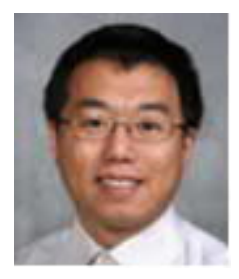

Xinyu Du received B.Sc. and M.Sc. degrees in automation from Tsinghua University, Beijing, China, in 2001 and 2004, respectively, and a Ph.D. in electrical engineering from Wayne State University, MI, USA, in 2012. He has been working at General Motors Global R\&D Center, Warren, MI, since 2010, and currently holds the staff researcher position in the vehicle systems research lab. His research interests include fuzzy hybrid system, vehicle health management, deep learning and data analytics. He has published more than 30 peer review papers and holds 33 patents or patent applications. He has been serving as an associate editor for Journal of Intelligent and Fuzzy Systems from 2012 and IEEE Access from 2018. He received the Boss Kettering Award from General Motors for his contribution in integrated starting system prognosis in 2015.

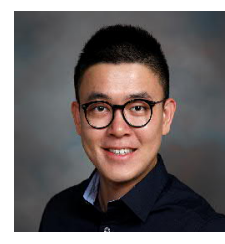

Lichao Mai received dual B.S. degree in automotive engineering from Wuhan University of Technology, Wuhan, China, in 2016, and in mechanical engineering from the University of Missouri, MO, USA, in 2016, respectively. He received a M.S. degree in mechanical engineering from the University of Texas at Austin, TX, USA, in 2018. He has been working at General Motors Global R\&D Center, Warren, MI, since 2018. His research interests include vehicle health management, autonomous driving and data analytics.

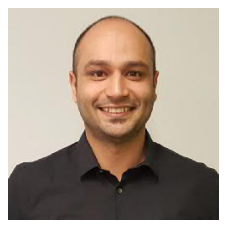

Hamed Kazemi received his Ph.D. degree in biomedical engineering from McGill University, Canada in 2015, and M.Eng degree in mechanical engineering from National University of Singapore in 2009 and B.Sc. degree in mechanical engineering from Tehran Polytechnique in 2006. He has been working at General Motors, Canadian Technical Center, Markham, ON, since 2018, as Controls Development Engineer in the Advanced Vehicle Prognostics team. Prior to joining General Motors, he has worked on developing collaborative robotic arms, rehabilitation robotics and haptic devices and received two consecutive awards for excellence in research from Quebec government (FRSQ). His research interests include robotics, autonomous systems and control.

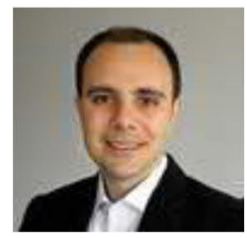

Hossein Sadjadi received his Ph.D. degree in electrical engineering from Queen's University, Canada, and M.Sc. degree in mechatronics and B.Sc. degree in electrical engineering from the American University of Sharjah, UAE. He has been working at General Motors, Canadian Technical Center, Markham, ON, since 2017, and is currently the Engineering Lead and Feature Owner for Vehicle Health Management. He also had served as a post-doctoral medical robotic researcher at Queen's university, senior automation engineer for industrial Siemens SCADA/DCS solutions, and senior mechatronics specialist at AUS mechatronics center. His research interests include autonomous systems and medical robotics. He has published numerous patents and articles in these areas, featured at IEEE transactions journals, and received several awards. 